\title{
Comparisons of the inter-joint coordination between leading and trailing limbs when crossing obstacles of different heights
}

\author{
Tung-Wu Lu ${ }^{\mathrm{a}, *}$, Hsiao-Ching Yen ${ }^{\mathrm{a}, \mathrm{b}}$, Hao-Ling Chen ${ }^{\mathrm{a}, \mathrm{c}}$ \\ a Institute of Biomedical Engineering, National Taiwan University, Taiwan \\ ${ }^{\mathrm{b}}$ Department of Physical Medicine and Rehabilitation, National Taiwan University Hospital, Taiwan \\ ${ }^{\mathrm{c}}$ School of Occupational Therapy, National Taiwan University, Taiwan
}

Received 8 November 2006; received in revised form 21 March 2007; accepted 15 April 2007

\begin{abstract}
Fifteen normal adults walked and crossed obstacles of different heights (10\%, 20\% and 30\% of leg length) with each limb while kinematic data were measured to obtain joint angles in the sagittal plane. Phase angles of each joint were calculated from the angular velocities $\left(x^{\prime}\right)$ and displacements $(x)$ as $\varphi=\tan ^{-1}\left(x^{\prime} / x\right)$. Relative phase angles were then calculated by subtracting phase angles of a distal joint from the proximal joint $\left(\varphi_{\text {hip-knee }}, \varphi_{\text {knee-ankle }}\right)$. The standard deviations of the relative phase curve points for the stance and swing phase for each obstacle height were averaged to obtain the respective deviation phase (DP) values. The calculated DP variables were tested using a two-factor repeated ANOVA. The leading and trailing limbs were found to have similar patterns of inter-joint coordination, but different levels of stability, the leading being more stable than the trailing during swing $(p<0.05)$, while only leading knee-ankle coordination was less stable than that of the trailing during stance $(p<0.05)$. Only the stability of the knee-ankle coordination for both limbs decreased with increasing obstacle height during stance $(p<0.05)$. It is suggested that clinical obstacle-crossing training programs for patients with unilateral pathology should include the training of the affected limb, not only as leading but also as trailing limb. An increase of the stability of the ankle joint may be helpful for the stability of the knee-ankle coordination and thus for the general performance of obstacle-crossing.
\end{abstract}

(C) 2007 Elsevier B.V. All rights reserved.

Keywords: Gait; Obstacles; Coordination; Relative phase; Phase plot; Stability

\section{Introduction}

Obstacle-crossing during walking is a complex motor task, requiring precise swing foot control while maintaining body balance through highly coordinated joint movements of the stance and swing limbs [1-3]. Failure to do this may result in tripping over obstacles, one of the most frequent causes of falls in the elderly [1,3-6]. Because of the requirements on multi-joint coordination and end-point control, obstacle-crossing has also been included in Frenkel's exercises for balance and coordination training for neurological patients [7]. Stability of the coordination patterns is a fundamental feature of consistent functional motions $[8,9]$. Therefore, data on the stability of the inter-

\footnotetext{
* Corresponding author. Tel.: +886 2 33653335; fax: +886 233653335.

E-mail address: twlu@ntu.edu.tw (T.-W. Lu).
}

joint coordination will be helpful for a better understanding of the control of the locomotor system during obstaclecrossing. This knowledge will be useful for the development of fall-prevention strategies and for their efficient use in patient rehabilitation.

Previous studies on the mechanics and control of the lower extremities during obstacle-crossing were done mainly through kinematic and kinetic analyses of crossing obstacles of different heights [1-3,7,10-12]. Emphasis has been on foot trajectory descriptions using variables, such as foot clearance, foot-obstacle horizontal distances and joint angles and moments. These studies reported the changes of the states of individual joints, but the data failed to reveal multi-joint coordination performance. To address these issues, Lu et al. used end-point variables combined with joint crossing angles to study multi-joint coordination patterns at the instant when the foot was above the obstacle 
[1]. However, the coordination patterns for the whole cycle are not available, although inter-segmental coordination patterns for the whole cycle during various other activities can be found in previous studies $[8,9,13-16]$.

Whereas inter-segmental coordination is defined as the relationship between two segments, and affects only the motion of one joint, inter-joint coordination is the relationship between the motions of two joints, including angular positions and velocities which are associated with information from afferent joint receptors [17]. Thus, inter-joint coordination may provide more information on how the central nervous system (CNS) organizes the various joints to perform functional activities. Previous studies suggest that apart from joint angular positions, angular velocities of the swing joints are also important in controlling obstaclecrossing $[18,19]$. Several methods have been proposed to study inter-joint coordination, including angle-angle plots, principal component analysis and relative phase plots $[17,20]$. Only the method of relative phase plots combines information on joint angular positions and velocities. The method of phase plots has been used to gain insight into the behavior of non-linear, limit cycle oscillatory systems in engineering. Since ensembles of non-linear, limit cycle oscillatory processes are used to characterize biological systems, their properties via relative phase plots are used to predict the patterns of inter-limb [21,22] and inter-joint coordination [17]. The relative phase plot of two joints was based on data from phase plots of each joint to reveal the control processes of the joints [17,23]. Relative phases between two joints have been used to study the coupling of two joints during a multi-joint movement [17], while the variability of the relative phase plots of repeated trials was used to quantify the stability of the inter-joint coordination patterns during the movement $[13,17,24]$. Therefore, the method of relative phase plot can be very useful in studying the patterns and stability of inter-joint coordination of the locomotor system during the whole cycle of multi-joint movements, including obstacle-crossing.

Studies on inter-joint coordination have been on the upper extremity $[25,26]$ or trunk and hip $[27,28]$, but few have focused on the lower limbs. Lindbeck and Kjellberg were among the few who studied gender differences in interjoint coordination when lifting a box with handles [27]. However, no study has investigated both the patterns and stability of inter-joint coordination of the lower limbs when walking over obstacles. Moreover, it remains unclear whether these characteristics would be different between the leading and trailing limbs. Previous studies suggested that different kinematic strategies were adopted by leading and trailing limbs when crossing obstacles with different heights $[1,2,10]$. A factor that may contribute to these differences is that the leading foot can be visually guided whereas the trailing foot cannot [10]. In addition, their biomechanical demands are also different [3]. Therefore, knowledge of the differences between the leading and trailing limbs in the pattern and stability of inter-joint coordination may help in the design of fall-prevention methods and in the rehabilitation of patients with unilateral pathology during obstacle-crossing.

The purposes of this study were thus to determine and compare the patterns and stability of the inter-joint coordination between the leading and trailing limbs during obstacle-crossing, and to study the effects of obstacle heights on these features of inter-joint coordination.

\section{Materials and methods}

Fifteen healthy young adults (age: $23 \pm 3$ years, height: $172.1 \pm 6.3 \mathrm{~cm}$ and mass: $68 \pm 8.6 \mathrm{~kg}$ ) participated with informed consent. They had corrected-to-normal vision and were free of neuromusculoskeletal pathology that might affect gait and/or cognitive function. Permission to conduct the study was provided by the Institutional Human Research Ethics Committee.

In a gait laboratory, each subject walked at a self-selected pace on an 8-m walkway and crossed a height-adjustable obstacle composed of a $1.5 \mathrm{~m}$ long aluminum tube placed across a metal frame. Two markers were placed on either end of the tube to define the obstacle position. Twenty-eight markers were used to track the motion of the body segments, including the pelvis (ASISs and PSISs), as well as each thigh (greater trochanter, mid-thigh, medial and lateral epicondyles), shank (head of fibula, tibial tuberocity, medial and lateral malleolus) and foot (navicular tuberosity, fifth metatarsal base, big toe and heel). Kinematic data were measured using a 7-camera motion analysis system (Vicon 512, Oxford Metrics Group, UK). Test conditions included crossing obstacles of three different heights $(10 \%, 20 \%$ and $30 \%$ of leg length) for both limbs. Six trials, three for each leg, for each condition were obtained.

Each body segment was embedded with an orthogonal coordinate system with the positive $x$-axis directed anteriorly, positive $y$ axis superiorly and positive $z$-axis to the right. A Cardanic rotation sequence $(\mathrm{Z}-\mathrm{X}-\mathrm{Y})$ was used to describe the rotational movements of each joint. The crossing stride (CS) of the leading limb began at toe-off before the obstacle and ended at the next toe-off, while that for the trailing limb was defined as heelstrike before the obstacle to the next heelstrike. For the angles in the sagittal plane angular velocities were calculated for each joint using the generalized cross-validatory spline method [29]. Phase plots of angular velocities against angular displacements for each joint were then generated. Angular displacements were normalized such that the range of angular positions during movement lay between -1 and 1 , with the midpoint located at zero. Angular velocity values were normalized by the maximum absolute velocity during the movement $[8,9,23]$. The phase angle $(\varphi)$ was obtained from the normalized phase plot of each CS as the angle formed between a line from the origin $(0,0)$ to the current data point $\left(x, x^{\prime}\right)$ and the right horizontal (Fig. 1), or calculated using $\varphi=\tan ^{-1}\left(x^{\prime} / x\right)$ [15,23]. The calculated phase angles were in the range of $0-180^{\circ}$, with positive values in the first and second quadrants, and negative values in the third and fourth (Fig. 1). Relative phase angles (RPA) between two adjacent joints were then calculated by subtracting the phase angle of the distal joint from that of the proximal, namely $\varphi_{\text {hip-knee }}$ and

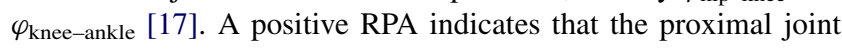
leads the distal, while a negative one means the reverse. If an RPA is close to $0^{\circ}$ or $\pm 360^{\circ}$, the two joints are moving in a similar fashion 


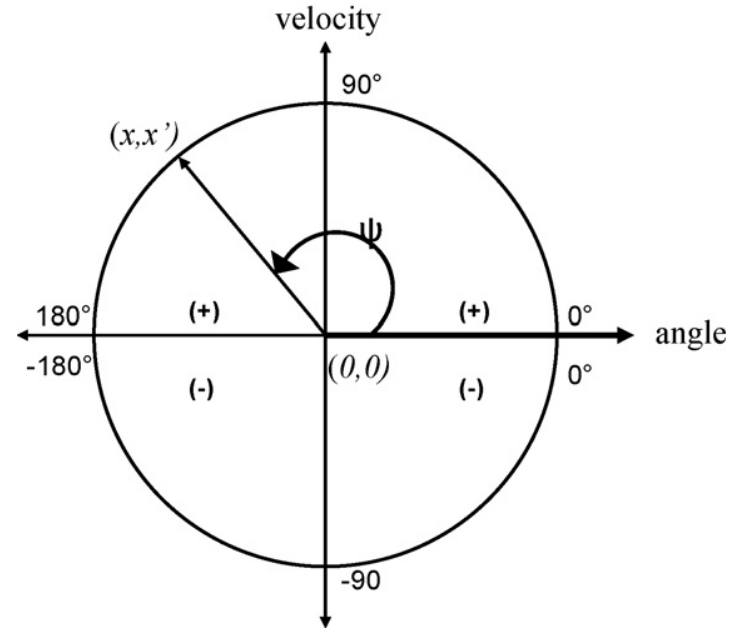

Fig. 1. Definition of the phase angle in the phase plot.

or in-phase. If an RPA is close to $\pm 180^{\circ}$, the two joints are moving in an opposite fashion or out-of-phase. For each inter-joint relationship, the RPA curves from the six trials of all subjects were ensemble-averaged for each obstacle height condition to reveal the general patterns of the inter-joint coordination. To quantify the stability of the inter-joint coordination, each RPA curve was first represented by 101 points and an ensemble RPA curve from the six trials for each subject was obtained. A parameter called deviation phase (DP) was then calculated by averaging the standard deviations of the ensemble RPA curve points for the stance and swing phase for each obstacle height $[8,9]$. A low DP value indicates a more stable relationship between the two joints $[8,9,30]$.

The effects of the limb (leading and trailing) and height on the calculated DP variables were tested using two-factor repeated measures ANOVA. If there were significant interactions between the main effects of limb and height, multiple comparisons were performed. If a height effect was found, a polynomial test was performed to determine the trend (linear or quadratic). A significance level of 0.05 was set for all statistical tests. All statistical analyses were conducted with SPSS (Version 11, Chicago, IL).

\section{Results}

The ensemble-averaged phase plots of the leading and trailing joints for all conditions are shown in Fig. 2. For both limbs, the hip and knee trajectories were all in forms of nearly closed and period circles, while those for the ankle had a different form of trajectories with varying amplitude. According to Kelso et al., these phase states were all stable [22]. These trajectories were almost the same for all obstacle heights, although small differences existed around heelstrike for the leading limb and for the ankle joint (Fig. 2).

The ensemble-averaged hip-knee $\left(\varphi_{\text {hip-knee }}\right)$ and kneeankle $\left(\varphi_{\text {knee-ankle }}\right)$ RPA for leading and trailing limbs for all conditions are shown in Fig. 3. Under all conditions, the leading hip-knee RPA curves remained around $+40^{\circ}$ during the swing phase, but showed a $\mathrm{W}$-shape with two negative peaks of about $-130^{\circ}$ and $-325^{\circ}$ during the stance phase
(Fig. 3A). The curves returned to around $+40^{\circ}$ at the end of the stance phase. Similar shapes were also found for the leading knee-ankle RPA curves, except when the RPA increased to around $+220^{\circ}$ at approximately $80 \% \mathrm{CS}$ and remained almost unchanged thereafter (Fig. 3B). During the leading swing phase, the hip-knee and knee-ankle rotations were all in-phase. When the trailing toe was above the obstacle, the leading hip and knee tended to rotate in-phase for all conditions, while the motion of the leading knee and ankle were almost out-of-phase.

The trailing hip-knee RPA curves remained within $+50^{\circ}$ during the swing phase for all conditions, but had two negative peaks (approximately $-227^{\circ}$ and $-342^{\circ}$ ), and one positive peak (about $50^{\circ}$ ) during the stance phase (Fig. 3C). Although the trailing knee-ankle RPA curves remained at around $-40^{\circ}$ during the swing phase, their shapes were similar to those of the hip-knee, but with reversed signs during the stance phase (Fig. 3D). When the leading toe was above the obstacle, the motions of the trailing hip and knee were in-phase under all conditions, but those of the trailing knee and ankle were out-of-phase. This trend was similar for the leading limb RPA curves. During stance phase, the trailing limb RPA curves had several short plateau periods, but none for the leading limb, indicating that the trailing limb RPA remained at the peak value for longer than for the leading limb.

The DP values of the leading and trailing RPA curves (hip-knee and knee-ankle) during the swing and stance phase are shown in Tables 1 and 2. There were no significant interactions between the limb and height effects. During swing phase, the DP values of the leading hip-knee and knee-ankle RPA curves were significantly smaller than those for the trailing limb, which were not affected by obstacle height. During stance phase, the DP values of the leading hip-knee RPA were not significantly different from those of the trailing RPA $(p>0.05)$, while those of the leading knee-ankle RPA were significantly greater than those of the trailing RPA ( $p<0.05$; Table 2). There was no significant height effect on DP values of the hip-knee RPA, but the DP values of the knee-ankle RPA increased linearly with increasing obstacle height $(p<0.05$; Table 2$)$.

\section{Discussion}

The patterns of the joint phase plots suggest that the intersegment coordination for each limb joint was stable and largely independent of obstacle height during obstaclecrossing. Therefore, insight into the overall control of the leading and trailing limbs requires a closer examination of the data on inter-joint coordination. During swing phase, the hip and knee joints were moving nearly in-phase or in synchronization for both limbs. The knee and ankle joints also showed nearly in-phase patterns. During stance phase, however, for both limbs, the inter-joint coordination between the hip and knee, as well as knee and ankle were 

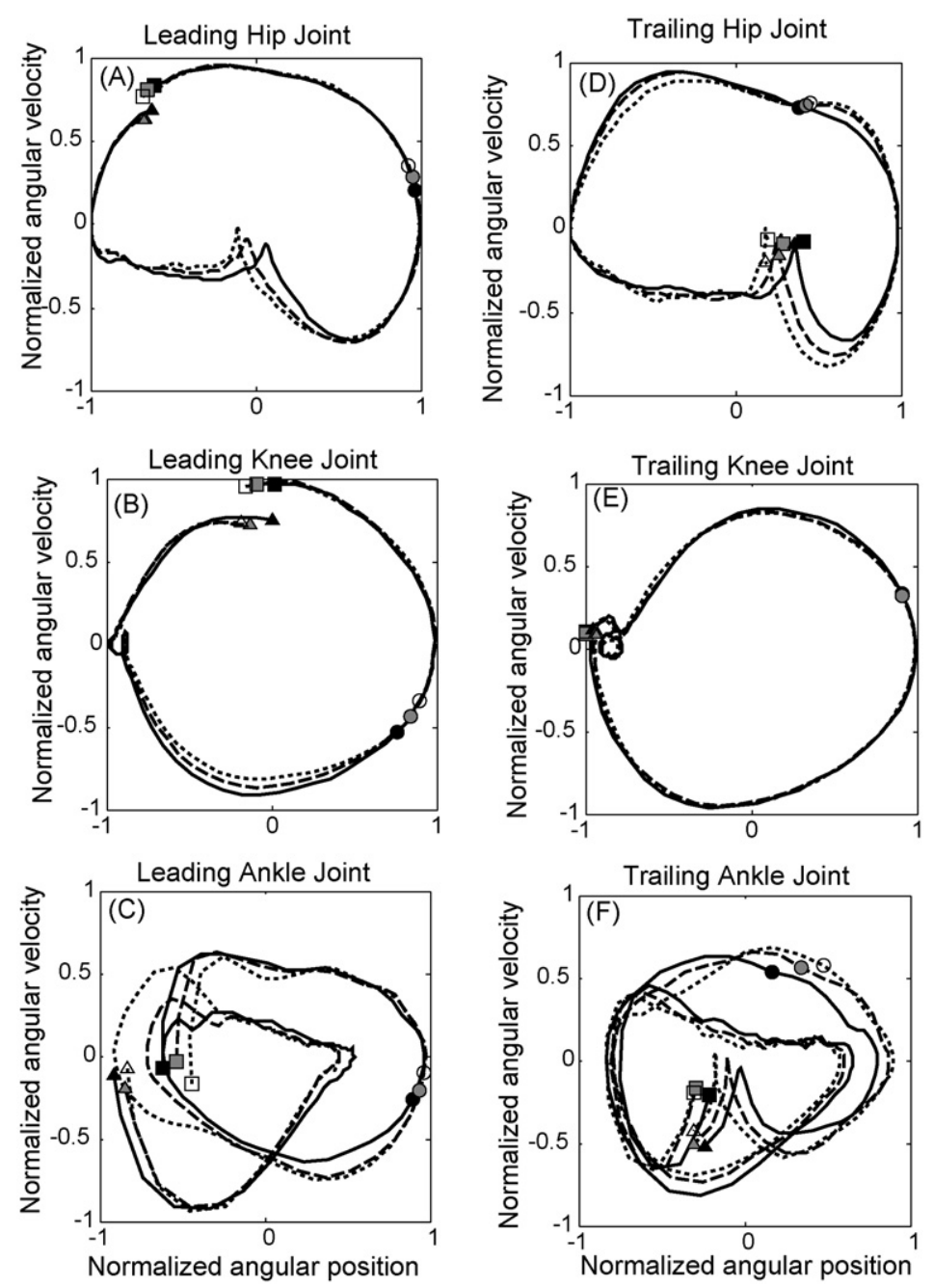

Fig. 2. The ensemble-averaged phase plots of the hip, knee and ankle for the leading (A-C) and trailing (D-F) limbs when crossing obstacles of $10 \%$ (solid lines, black markers), 20\% (dashed lines, grey markers) and 30\% (dotted lines, white markers) of leg length. Square markers indicate beginning of crossing cycle, circular markers the crossing position and triangular markers end of crossing cycle.

switching between in-phase and out-of-phase patterns throughout the period. A difference between the patterns of the leading and trailing RPA curves during stance phase was the short plateau periods in the trailing RPA curves. When the trailing toe was above the obstacle, the in-phase hip-knee and out-of-phase knee-ankle coordination patterns in the leading stance limb suggested that the ankle might play an important role in modulating the stability of body. A similar phenomenon was also found in the trailing limb when the leading toe was above the obstacle. The mechanisms behind these phenomena may be similar to the ankle strategy used in maintaining balance as a singlesegment inverted pendulum by producing a torque at the ankle during quiet standing [31-33]. Since obstacle-crossing requires precise swing foot control, while body balance is maintained mainly by the stance limb, the difference of the inter-joint coordination patterns between the swing and stance phases further supports the idea that well-controlled inter-limb coordination is needed to perform stable and safe obstacle-crossing. A better understanding of this inter-limb coordination requires knowledge of the control of leading and trailing limbs individually. Since both limbs had inphase patterns during swing phase and switched between inphase and out-of-phase patterns during stance phase, and the similarity was not affected by obstacle height, further examination of the stability of the inter-joint coordination between the limbs is necessary.

During stance phase the leading hip-knee coordination was as stable as that of the trailing limb, while the leading knee-ankle coordination was less stable than that of the trailing limb (Table 2). This may be due to the difference of mechanical demands between the two limbs. When the leading limb was crossing and the trailing limb was supporting the body, the body's center of mass (COM) was moving away from the base of support (the trailing limb). When the trailing limb was crossing, the COM was moving towards the leading stance foot. Thus, more stable trailing knee-ankle coordination may be helpful for the greater demand on the trailing stance limb to maintain whole body balance. 
(A) Leading Hip-Knee Coordination

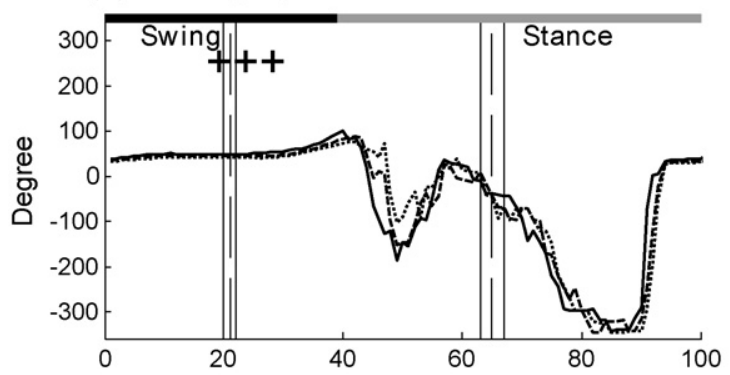

(B) Leading Knee-Ankle Coordination

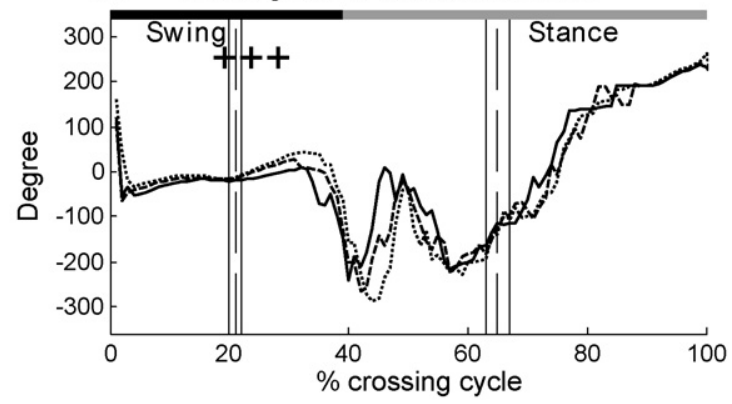

(C) Trailing Hip-Knee Coordination

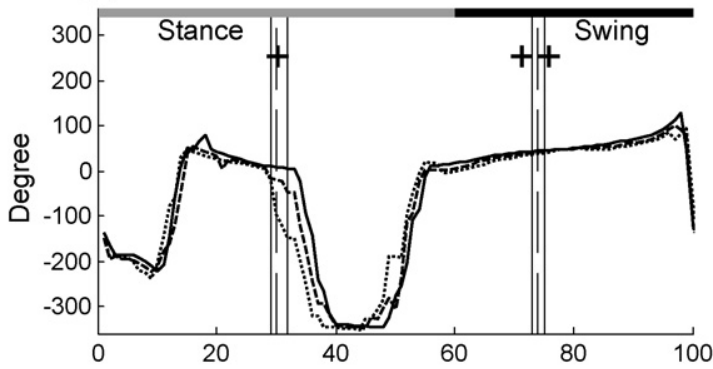

(D) Trailing Knee-Ankle Coordination

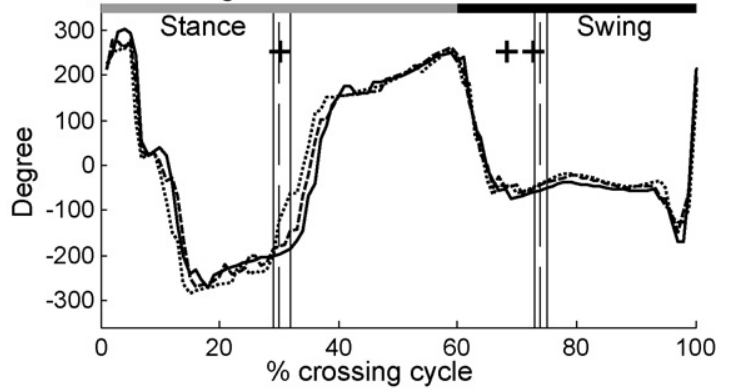

Fig. 3. The ensemble-averaged relative phase angles of the hip-knee and knee-ankle coordination for the leading (A-B) and trailing (C-D) limbs when crossing obstacles of $10 \%$ (solid lines), $20 \%$ (dashed lines) and 30\% (dotted lines) of leg length. Symbol ' +' indicates leading swing toe crossing and ' ++ ' trailing swing toe crossing.

During swing phase, the leading hip-knee and kneeankle coordination was more stable than that of the trailing limb (Table 1). This difference may be associated with the lack of visual feedback about the time-varying relationship between the trailing limb and the obstacle, increasing the difficulty in the control stability of the trailing swing limb inter-joint coordination. The more stable inter-joint coordination in the trailing stance limb might also contribute to better stability of the leading swing limb inter-joint coordination, as the stability of the inter-joint coordination of the stance limb was a prerequisite for the swing limb stability during obstacle-crossing. More stable inter-joint coordination in both limbs, when the leading was crossing, seemed necessary for maintaining whole body balance with accurate foot clearance when the COM was moving away from the stance limb. The relatively reduced stability of the inter-joint coordination of both limbs when the trailing limb was crossing did not seem to present a serious problem as the COM was moving towards the leading stance foot and whole body stability was easier to achieve.
From the findings of the present study, it is evident that different requirements of inter-joint coordination stability are needed between the leading and trailing limbs. Clinical obstacle-crossing training programs for patients with unilateral pathology should thus include the training of the affected limb, not only as leading, but also as trailing limb. For safety considerations, these patients may lead with the affected side during obstacle-crossing in the early recovery stage.

During stance phase, only the stability of the knee-ankle coordination for both limbs decreased with increasing obstacle height. Since the body's COM height increased with increasing obstacle height for both limbs [3], more stable knee-ankle coordination would be helpful for the greater demand on the stance limb to maintain whole body balance. Therefore, decreased stability of the knee-ankle coordination may indicate an increase in difficulty in the control of the ankle joint in modulating the stability of the body. These results suggest that an increase in the stability of the ankle joint, achieved through training of both limbs by crossing obstacles of different heights, may be helpful for

Table 1

Means and standard deviations of the deviation phase (DP) values of the hip-knee and knee-ankle coordination during swing phase

\begin{tabular}{|c|c|c|c|c|c|c|c|c|}
\hline \multirow[t]{3}{*}{ DP value $\left(^{\circ}\right)$} & \multicolumn{6}{|c|}{ Obstacle height } & \multirow{3}{*}{$\begin{array}{l}\text { Limb and height } \\
\text { effect } p \text {-value }\end{array}$} & \multirow[t]{3}{*}{ Trend } \\
\hline & \multicolumn{2}{|l|}{$10 \%$} & \multicolumn{2}{|l|}{$20 \%$} & \multicolumn{2}{|l|}{$30 \%$} & & \\
\hline & Leading & Trailing & Leading & Trailing & Leading & Trailing & & \\
\hline Hip-knee & $6.49(2.43)$ & $19.60(10.44)$ & $5.16(1.89)$ & $18.01(7.05)$ & $5.75(1.79)$ & $15.18(7.38)$ & $p_{1}<0.001^{*}, p_{\mathrm{h}}=0.259$ & - \\
\hline Knee-ankle & $26.57(9.81)$ & $51.02(14.86)$ & $31.26(13.36)$ & $55.07(14.26)$ & $32.43(10.25)$ & $57.79(11.50)$ & $p_{1}<0.001^{*}, p_{\mathrm{h}}=0.102$ & - \\
\hline
\end{tabular}

$p_{1}$ represents a main limb effect and $p_{\mathrm{h}}$ a main height effect. Symbol (-) indicates no effect.

* Significant main effect (i.e., $p<0.05$ ). 
Table 2

Means and standard deviations of the deviation phase (DP) values of the hip-knee and knee-ankle coordination during stance phase

\begin{tabular}{|c|c|c|c|c|c|c|c|c|}
\hline \multirow[t]{3}{*}{ DP value $\left({ }^{\circ}\right)$} & \multicolumn{6}{|l|}{ Obstacle height } & \multirow{3}{*}{$\begin{array}{l}\text { Limb and height } \\
\text { effect } p \text {-value }\end{array}$} & \multirow[t]{3}{*}{ Trend } \\
\hline & \multicolumn{2}{|l|}{$10 \%$} & \multicolumn{2}{|l|}{$20 \%$} & \multicolumn{2}{|l|}{$30 \%$} & & \\
\hline & Leading & Trailing & Leading & Trailing & Leading & Trailing & & \\
\hline Hip-knee & $80.98(24.13)$ & $65.13(13.94)$ & $77.88(27.01)$ & $68.65(14.99)$ & $72.69(18.75)$ & $81.00(26.30)$ & $p_{1}=0.247 p_{\mathrm{h}}=0.435$ & - \\
\hline Knee-ankle & $81.18(22.87)$ & $59.80(13.00)$ & $82.12(24.54)$ & $62.38(11.90)$ & $85.42(20.19)$ & $79.89(26.54)$ & $p_{1}=0.009^{*} p_{\mathrm{h}}=0.020^{*}$ & $\uparrow$ \\
\hline
\end{tabular}

$p_{1}$ represents a main limb effect and $p_{\mathrm{h}}$ a main height effect. With increasing obstacle heights, $(\uparrow)$ indicates a significant linearly increasing trend, $(\downarrow)$ a significant linearly decreasing trend and (-) no effect.

* Significant main effect (i.e., $p<0.05$ ).

the stability of knee-ankle coordination, and thus for the general performance of obstacle-crossing.

In the current study, data were obtained for crossing obstacles less than $30 \%$ leg length in height because obstacles higher than $30 \%$ leg length are rare in daily life. The results should apply to most daily conditions although caution should be exercised when generalizing the results to higher obstacles. The present study was limited to stability and patterns of inter-joint coordination in the sagittal plane. Further study may include analysis in other planes.

\section{Conclusions}

During obstacle-crossing the leading and trailing limbs were found to have similar patterns of inter-joint coordination, but with different levels of stability, which may be associated with the difference in mechanical demands during stance and the lack of visual feedback about the time-varying relationship between the trailing limb and the obstacle during swing. It is suggested that clinical obstacle-crossing training programs for patients with unilateral pathology should include the training of the affected limb, not only as leading, but also as trailing limb. An increase in the stability of the ankle joint may be helpful for the stability of the knee-ankle coordination, and thus for the general performance of obstacle-crossing.

\section{Conflicts of interest}

We, Tung-Wu Lu, Hsiao-Ching Yen and Hao-Ling Chen, declare that we have no proprietary, financial, professional or other personal interest of any nature or kind in any product, service and/or company that could be construed as influencing the position presented in or the view of the manuscript entitled "Comparisons of the inter-joint coordination between leading and trailing limbs when crossing obstacles of different heights".

\section{Acknowledgement}

The authors gratefully acknowledge the financial support from the National Science Council of Taiwan (NSC95-2314B002-167-MY2).

\section{References}

[1] Lu TW, Chen HL, Chen S-C. Comparisons of the lower limb kinematics between young and older adults when crossing obstacles of different heights. Gait Posture 2006;23:471-9.

[2] Chen HL, Lu TW, Lin HC. Three-dimensional kinematic analysis of stepping over obstacles in young subjects. Biomed Eng Appl Basis Commun 2004; 16:157-63.

[3] Chen HL, Lu TW. Comparisons of the joint moments between leading and trailing limb in young adults when stepping over obstacles. Gait Posture 2006;23:69-77.

[4] Tinetti M, Speechley M, Ginter S. Risk factors for falls among elderly persons living in the community. N Eng J Med 1988;319:170-7.

[5] Tinetti M, Speechley M. Prevention of falls among the elderly. N Eng J Med 1989;320:1055-9.

[6] Blake A, Morgan M, Dallosso H, Ebrahim S, Arie T, Fentem P, et al. Falls by elderly people at home: prevalence and associated factors. Age Aging 1988;17:365-72.

[7] Umphred DA, Jewell MJ. Neurological rehabilitation. St. Louis: C.V. Mosby Co.; 1995.

[8] Stergiou N, Scholten SD, Jensen JL, Blanke D. Intralimb coordination following obstacle clearance during running: the effect of obstacle height. Gait Posture 2001;13:210-20.

[9] Stergiou N, Jensen JL, Bates BT, Scholten SD, Tzetzis G. A dynamical systems investigation of lower extremity coordination during running over obstacles. Clin Biomech 2001;16:213-21.

[10] Sparrow W, Shinkfield AJ, Chow S, Begg R. Characteristics of gait in stepping over obstacles. Hum Mov Sci 1996;15:605-22.

[11] Patla A, Rietdyk S. Visual control of limb trajectory over obstacles during locomotion: effect of obstacle height. Gait Posture 1993;1:45-60.

[12] Chou LS, Draganich LF. Stepping over an obstacle increases the motions and moments of the joints of the trailing limb in young adults. J Biomech 1997;30:331-7.

[13] van Uden CJ, Bloo JK, Kooloos JG, van Kampen A, de Witte J, Wagenaar RC. Coordination and stability of one-legged hopping patterns in patients with anterior cruciate ligament reconstruction: preliminary results. Clin Biomech 2003;84-7.

[14] Piek JP, Gasson N, Barrett N, Case I. Limb and gender differences in the development of coordination in early infancy. Hum Mov Sci 2002;21:621-39.

[15] Li L, van den Bogert ECH, Caldwell GE, van Emmerik REA, Hamill J. Coordination patterns of walking and running at similar speed and stride frequency. Hum Mov Sci 1999;18:67-85.

[16] Clark JE, Phillips SJ. A longitudinal study of intralimb coordination in the first year of independent walking: a dynamical systems analysis. Child Dev 1993;64:1143-57.

[17] Burgess-Limerick R, Abernethy B, Neal RJ. Relative phase quantifies interjoint coordination. J Biomech 1993;26:91-4.

[18] Patla A, Prentice S. The role of active forces and intersegmental dynamics in the control of limb trajectory over obstacles during locomotion in humans. Exp Brain Res 1995;106:499-504.

[19] Austin GP, Garrett GE, Bohannon RW. Kinematic analysis of obstacle clearance during locomotion. Gait Posture 1999;10:109-20. 
[20] Cirstea MC, Mitnitski AB, Feldman AG. Interjoint coordination dynamics during reaching in stroke. Exp Brain Res 2003;151: 289-300.

[21] Schoner G, Kelso JAS. Dynamic pattern generation in behavioral and neural systems. Science 1988;239:1513-20.

[22] Kelso JAS, Holt KG, Rubin P, Kugler PN. Patterns of human interlimb coordination emerge from the properties of non-linear, limit cycle oscillatory processes: theory and data. J Mot Behav 1981;13:226-61.

[23] Kurz MJ, Stergiou N. Effect of normalization and phase angle calculations on continuous relative phase. J Biomech 2002;35:369-74.

[24] Hamill J, van Emmerik REA, Heiderscheit BC, Li L. A dynamical systems approach to lower extremity running injuries. Clin Biomech 1999;14:297-308.

[25] Meulenbroek RG, Thomassen AJ, van Lieshout PH, Swinnen SP. The stability of pen-joint and interjoint coordination in loop writing. Acta Psychol 1998;100:55-70.

[26] Dounskaia N, Van Gemmert AW, Stelmach GE. Interjoint coordination during handwriting-like movements. Exp Brain Res 2000;135: $127-40$.
[27] Lindbeck L, Kjellberg K. Gender differences in lifting technique. Ergonomics 2001;44:202-14.

[28] van Emmerik REA, Wagenaar RC. Effects of walking velocity on relative phase dynamics in the trunk in human walking. J Biomech 1996;29:1175-84.

[29] Woltring HJ. A FORTRAN package for generalized, cross-validatory spline smoothing and differentiation. Adv Eng Software 1986;8: 104-13.

[30] Riley MA, Santana M-V, Turvey MT. Deterministic variability and stability in detuned bimanual rhythmic coordination. Hum Mov Sci 2001;20:343-69.

[31] Karlsson A, Lanshammar H. Analysis of postural sway strategies using an inverted pendulum model and force plate data. Gait Posture 1997;5:198-203.

[32] Gatev P, Thomas S, Kepple T, Hallett M. Feedforward ankle strategy of balance during quiet stance in adults. J Physiol 1999;514:915-28.

[33] Colobert B, Cretual A, Allard P, Delamarche P. Force-plate-based computation of ankle and hip strategies from double-inverted pendulum model. Clin Biomech 2006;21:427-34. 